\title{
AUTHOR INDEX Volume 2
}

Angelika C. Hailer \& Siegfried M. Rump, Evaluation of hedge effectiveness tests

Arnaud De Toytot, see Sue Harding

Bridget Gandy, Roger Merritt, Mark Oline, Joseph St. Denis \& William Mann, Hedge accounting and derivatives study for corporates disclosure, hedge accounting, and restatement risk

Bryan Mase, see Kyriacos Kyriacou

David M. Schizer, see Michael R. Powers

Davinder K. Malhotra, Mukesh Chaudhry \& Vivek Bhargava, Structural relationships between semiannual and annual swap rates

Don M. Chance \& Tung-Hsiao Yang, The utility-based valuation and cost of executive stock options in a binomial framework: Issues and methodologies

Duncan Campbell-Smith \& Peter Barnes-Wallis Rolls-Royce Group Plc: Transition from UK GAAP to International Financial Reporting Standards (IFRS)

Emmanuel Dubois-Pelerin, see Sue Harding

James A. Largay III, see Susan S. Hamlen

James N. Bodurtha, Jr., Divergent FAS-133 and IAS-39 interest rate risk hedge effectiveness: Problem and remedies
Joseph St. Denis, see Bridget Gandy

$1(2005) 31$

1 (2005) 111

Junning Cai, Accounting for employee stock options and mandatory expensing: An economics perspective

Kit Pong Wong, Operating leverage and the interaction between abandonment options and exotic hedging

Kiyohito Utsunomiya,

2 (2005) 231

2 (2005) 203

2 (2005) 155

Employee stock options in Japan: Determinants of their issuance, their potential impact on corporate profits, and their association with stock prices

Kyriacos Kyriacou \& Bryan Mase, Executive stock option exercises, tax, and the

1 (2005) 63 predictive ability of transaction value

Maria Lemos, see Sue Harding

Mark Oline, see Bridget

Gandy

Martin Shubik, see Michael

$2(2005) 165$

R. Powers

Michael R. Powers, David M. Schizer \& Martin Shubik, How taxes affect market price: The "Longs and Shorts" of discounting and
2 (2005) 247

1 (2005) 111

2 (2005) 215

$1(2005) 1$
Mukesh Chaudhry, see

Davinder K. Malhotra

Peter Barnes-Wallis, see

Duncan Campbell-Smith

Phelim Boyle \& Weidong Tian, Executive stock options:

A firm value approach

Rob Jones, see Sue Harding

Robert A. Jones, see Yan Wu
$2(2005) 231$

$2(2005) 137$

$1(2005) 87$

$1(2005) 97$

2 (2005) 203

1 (2005) 111

2 (2005) 231

$2(2005) 155$

2 (2005) 189

1 (2005) 111 
Roger Merritt, see Bridget Gandy

Siegfried M. Rump, see Angelika C. Hailer

Sofiane Aboura, Pricing $C A C$ 40 index options with stochastic volatility

Sue Harding, Arnaud De

Toytot, Emmanuel Dubois-Pelerin, Rob Jones \& Maria Lemos, Transition without tears: A five-point plan for IFRS disclosure from standard $\&$ poor's ratings services

Susan S. Hamlen \& James A. Largay III, Has SFAS 133 made derivatives reporting more transparent? A look at the Dow-Jones 30
2 (2005) 231

1 (2005) 31

1 (2005) 77

1 (2005) 111

2 (2005) 215
Tung-Hsiao Yang, see Don

M. Chance

Vivek Bhargava, see Davinder

K. Malhotra

Wei Li \& William W.

Stammerjohan, Empirical

analysis of effects of SFAS

No. 133 on derivative use and earnings smoothing

Weidong Tian, see Phelim Boyle

William Mann, see Bridget Gandy

William W. Stammerjohan, see Wei Li

Yan Wu \& Robert A. Jones, Valuing employee stock options with exogenous and endogenous early exercise
$1(2005) 15$

2 (2005) 189

2 (2005) 231

1 (2005) 15

2 (2005) 165

$1(2005) 63$

$1(2005) 53$ 